\title{
Análisis Energético y Económico de Sistemas Simples de Cogeneración
}

\author{
Miguel A. Lozano y José Ramos \\ Universidad de Zaragoza, Departamento de Ingeniería Mecánica, GITSE-I3A, C/ Maria de Luna s/n, \\ 50018 Zaragoza-España (e-mail: miguel.lozano@unizar.es, jose.ramos@unizar.es)
}

\begin{abstract}
Resumen
En este artículo se investigan el ahorro de energía y los aspectos económicos relacionados con el empleo de motores alternativos de combustión interna en plantas de cogeneración para edificios. Se definen criterios técnicos para caracterizar la operación anual de los sistemas simples de cogeneración que atienden demandas variables de calor. También se analiza el problema de determinar la capacidad de los motores a instalar tomando en consideración diferentes estrategias de operación. La metodología de análisis propuesta se aplica a una planta que satisface la demanda de agua caliente sanitaria y calefacción de un complejo residencial. Los resultados permiten comparar diversas estrategias de operación.
\end{abstract}

Palabras clave: cogeneración, análisis termodinámico, eficiencia energética, ahorro de energía

\section{Thermodynamic and Economic Analysis of Simple Cogeneration Systems}

\begin{abstract}
This paper investigates energy savings and economic aspects related to the use of internal combustion engines in cogeneration plants for buildings. Technical criteria that characterize the operation of simple cogeneration systems with variable thermal energy demands are defined. Also, the problem of finding the annual operational strategies for the variations of load demands and determining the sizes of cogeneration plants is analyzed. The proposed methodology is applied to the analysis of a plant that satisfies the requirements of hot water and heating of a residential area. The results allow to compare different operating strategies.
\end{abstract}

Keywords: cogeneration, CHP, thermodynamic analysis, energy efficiency, energy savings 


\section{INTRODUCCIÓN}

Se define como Cogeneración a la producción conjunta, en proceso secuencial, de energía mecánica o eléctrica y de energía térmica útil, a partir de la misma fuente de energía primaria. Normalmente, los sistemas de cogeneración se diseñan de modo que sean capaces de satisfacer la demanda térmica del consumidor, ya que en la mayor parte de los casos resulta inviable la compraventa de energía térmica a un agente económico externo. Por el contrario, sí se puede comprar energía eléctrica, e incluso venderla bajo ciertas condiciones. Si el sistema de cogeneración se diseña para satisfacer las necesidades de trabajo del centro consumidor, prescindiéndose del apoyo de las compañías eléctricas, se dice que es de Energía Total. En la literatura (Petchers, 2003) puede encontrarse una descripción más detallada de estos conceptos.

Cada día parece más evidente que el mundo no camina por una senda que conduzca a un futuro energéticamente sostenible. A pesar de las iniciativas acordadas por los gobiernos y de los esfuerzos de las empresas, las emisiones de $\mathrm{CO}_{2}$ aumentaron un $20 \%$ durante la última década. Un estudio reciente de la Agencia Internacional de la Energía (IEA, 2006), estima que las emisiones en 2050 duplicarán las actuales. Este escenario tan alarmante puede evitarse, en parte, utilizando la cogeneración para aumentar la eficiencia del suministro energético. De hecho, la potencia eléctrica instalada en sistemas de cogeneración para la industria ha crecido mucho en las últimas décadas produciendo grandes ahorros energéticos y económicos. Pero, en los países desarrollados, el potencial de la cogeneración en la industria ya ha sido explotado y por ello ahora los esfuerzos se dirigen al sector de edificios. Por ejemplo, en España menos del 5\% de la potencia eléctrica instalada en cogeneración corresponde al sector Residencial-Comercial, lo que supone, considerando sus enormes consumos de agua caliente sanitaria (ACS), calefacción y climatización, desperdiciar un enorme potencial de ahorro. Tres de los motivos más frecuentes son: i) la presencia de múltiples usuarios de los servicios energéticos que piensan que el aprovisionamiento individual les ofrece mayor garantía, ii) la baja capacitación técnica o ausencia de personal encargado de la gestión energética, y iii) la dificultad de establecer una configuración adecuada para la planta de servicios energéticos a causa de las grandes fluctuaciones temporales de los consumos.

Para aprovechar dicho potencial algunos países han promulgado decretos y tomado medidas que promueven el uso de la cogeneración y de la Calefacción y Refrigeración de Distrito (District Heating and Cooling, DHC). En la Unión Europea, la Directiva 2002/91/CE relativa a la eficiencia energética de los edificios informa: "El sector de la vivienda y de los servicios, compuesto en su mayoría por edificios, absorbe más del $40 \%$ del consumo final de energía en la Comunidad y se encuentra en fase de expansión, tendencia que previsiblemente hará aumentar el consumo de energía y, por lo tanto, las emisiones de $\mathrm{CO}_{2}$." Su artículo 5 impone: "En los edificios nuevos con una superficie útil total de más de $1000 \mathrm{~m}^{2}$, los Estados miembros velarán por que la viabilidad técnica, medioambiental y económica de sistemas alternativos como la cogeneración y la calefacción o refrigeración central o urbana se tengan en cuenta antes de que se inicie la construcción". Por otro lado, la Directiva 2004/8/CE relativa al fomento de la cogeneración reconoce: "En la actualidad en la Comunidad está infrautilizado el potencial de la cogeneración como medida para ahorrar energía." Esta directiva considera que la cogeneración es de alta eficiencia cuando el ahorro energético es superior al $10 \%$.

Los sistemas de cogeneración que atienden a los edificios (centros comerciales, hospitales, hoteles, etc.) y urbanizaciones están diseñados para satisfacer sus demandas de electricidad, agua caliente sanitaria y calefacción. La viabilidad económica de estos sistemas queda garantizada cuando la producción simultánea de trabajo y calor, sobre todo la de calor, está respaldada por una demanda efectiva durante muchas horas al cabo del año (Cardona y Piacentino, 2003). Si el factor de utilización de los módulos de cogeneración es alto, se obtendrán grandes ahorros económicos y cortos períodos de recuperación de la inversión. Esto ocurre en las instalaciones ubicadas en lugares de clima frío que demandan calor durante un gran número de horas al año para suministro de servicios de calefacción y agua caliente sanitaria. Por el contrario, en lugares de clima templado la demanda de calor es pequeña, se concentra en pocos meses y el ahorro económico es bajo, pues solo quedan dos opciones malas: i) un corto periodo anual de funcionamiento del motor, limitado por el aprovechamiento del calor residual, que dificulta recuperar la inversión con ahorro, o por contra ii) un elevado despilfarro de calor, limitado por la condición legal de autogenerador, que solo resultará 
económico bajo condiciones extraordinarias de precios de combustibles y electricidad. Una posible alternativa en estos casos es recurrir a la trigeneración (TriGeMed, 2003; Lozano et al., 2004b). El calor cogenerado se emplea en el verano para cubrir la demanda de aire acondicionado mediante enfriadoras de absorción incorporadas a las instalaciones de cogeneración.

Para obtener grandes ahorros de energía, a la par con una alta rentabilidad económica en los sistemas de cogeneración para edificios, debe prestarse una gran atención tanto a su dimensionado correcto como a las estrategias de operación a emplear. Una manera de abordar el problema es recurrir a programas complejos de optimización numérica (Yokohama et al., 1994; Lozano, 2001), pero también resulta útil una aproximación analítica (Lucas, 2000; Tchouate y Bolle, 2002; Cardona y Piacentino, 2003), como la que aquí se propone. En este trabajo se presenta un método de evaluación energética y económica de las instalaciones de cogeneración destinadas al suministro de servicios energéticos a grandes edificios y complejos urbanos. La metodología desarrollada consta de las siguientes etapas: i) caracterización de las prestaciones energéticas de los módulos de cogeneración, ii) descripción de la demanda de calor del centro consumidor; y iii) determinación de la capacidad de cogeneración a instalar y de la política de operación a seguir. La metodología se aplica en la fase de prediseño de una planta de cogeneración, de tamaño medio y basada en módulos de cogeneración con motores de gas, destinada a satisfacer la demanda de ACS y calefacción de un complejo residencial.

\section{CRITERIOS DE EFICIENCIA}

Para caracterizar los sistemas de cogeneración se deben definir un conjunto de parámetros que permitan: i) valorar las oportunidades de inversión, ii) seleccionar el sistema más adecuado, y iii) optimizar su operación una vez instalado. Suponiendo, para simplificar, que el sistema de cogeneración es una caja negra, como la de la Fig. 1, que consume $F$ unidades de energía de combustible (poder calorífico inferior), produce simultáneamente $W$ unidades de trabajo y $Q$ unidades de calor. Para un análisis energético de la operación convendrá expresar $F, W$ y $Q$ como flujos de energía en el mismo Sistema de Unidades y manejar los parámetros siguientes:

$$
\begin{array}{ll}
\text { Eficiencia eléctrica } & \alpha_{W} \equiv W / F \\
\text { Eficiencia térmica } & \alpha_{Q} \equiv Q / F \\
\text { Eficiencia global } & \eta \equiv(W+Q) / F \\
\text { Relación calor-trabajo } & \beta \equiv Q / W
\end{array}
$$

Nótese, sin embargo, que para caracterizar por completo el sistema basta conocer 3 variables independientes de entre las anteriores. Por ejemplo, pueden emplearse $W, \alpha_{w}$ y $\beta$. Otros parámetros interesantes para el análisis muestran la ventaja comparativa de los sistemas de cogeneración sobre los sistemas convencionales de aprovisionamiento de calor y trabajo. Para definirlos, se representa en la Fig. 2 la operación de un sistema convencional que produce las mismas cantidades de trabajo y calor que el sistema de cogeneración.

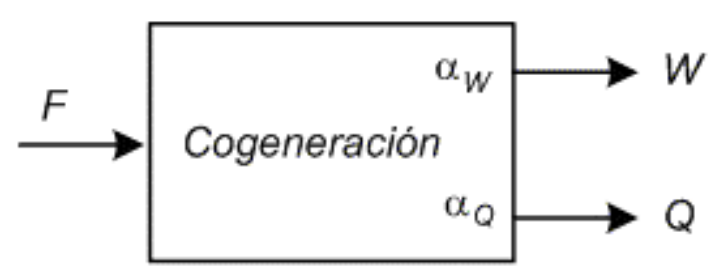

Fig. 1: Sistema de cogeneración

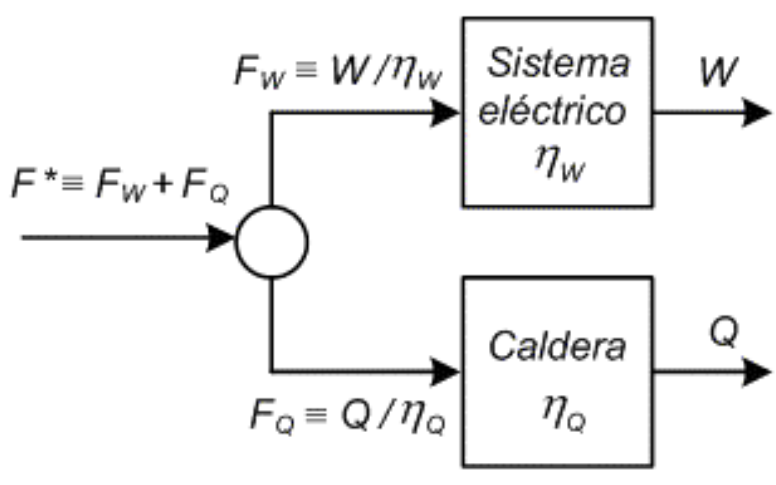

Fig. 2: Producción convencional 
Supóngase que se ha de suministrar $W$ y $Q$ para satisfacer las demandas del consumidor. Este debe decidir entre instalar un sistema de cogeneración o proceder de modo convencional comprando energía eléctrica a la compañía distribuidora (que se supone se ha producido con rendimiento $\eta_{w}$ ) e instalando una caldera de rendimiento $\eta_{Q}$ para producir el calor. La decisión a favor de la cogeneración conllevará un ahorro de combustible

$\Delta F \equiv F^{*}-F=\frac{W}{\eta_{W}}+\frac{Q}{\eta_{Q}}-F$

Relacionado con este concepto, se define el índice de ahorro de energía primaria IAF (Fuel Energy Saving Ratio, FESR), como el ahorro de combustible por unidad de energía requerida en el modo convencional de aprovisionamiento

$$
I A F \equiv \frac{\Delta F}{F^{*}}=1-\frac{F}{\frac{W}{\eta_{W}}+\frac{Q}{\eta_{Q}}}=1-\frac{1}{\frac{\alpha_{W}}{\eta_{W}}+\frac{\alpha_{Q}}{\eta_{Q}}}
$$

El último parámetro que se va a considerar es el rendimiento eléctrico equivalente, cuya definición proviene de utilizar un criterio particular a la hora de repartir el consumo de combustible entre los dos productos del sistema de cogeneración. Concretando, si una caldera convencional de rendimiento $\eta_{Q}$ consumiría el combustible $F_{Q}=Q / \eta_{Q}$ para producir (por obligación y sin cogenerar) el calor útil $Q$, la decisión de instalar (por interés económico) el sistema de cogeneración, supone un consumo adicional de combustible $F_{\text {eqw }}=F-F_{Q}$ para producir además la electricidad $W$. Por tanto, el rendimiento eléctrico equivalente es

$\eta_{e q} \equiv \frac{W}{F_{e q W}}=\frac{W}{F-Q / \eta_{Q}}=\frac{\alpha_{W}}{1-\alpha_{Q} / \eta_{Q}}$

Obsérvese como los criterios IAF y $\eta_{\text {eq }}$ ponen especial énfasis en averiguar si los sistemas de cogeneración transforman con mayor eficiencia la energía consumida en productos útiles (calor y trabajo) que los sistemas convencionales.

La disminución de emisiones de $\mathrm{CO}_{2}$ es un criterio ambiental que puede calcularse directamente a partir del ahorro de combustible en el caso de que este sea el mismo para cogeneración y para producción convencional. En caso de que los combustibles sean diferentes la disminución será

$\Delta C O_{2} \equiv c F_{W} \frac{W}{\eta_{W}}+c F_{Q} \frac{Q}{\eta_{Q}}-c_{F} F$

donde $c F_{i}$ es la emisión de $\mathrm{CO}_{2}$ por unidad de energía en el combustible $i$.

\section{SISTEMAS DE COGENERACION}

La mayor parte de las instalaciones de cogeneración en edificios utilizan motores de gas y algunas de ellas turbinas de gas. Martens (1998) y Li et al. (2006), han realizado un análisis estadístico de las prestaciones de estos motores. Las turbinas de vapor, microturbinas de gas, células de combustible, y otros equipos de producción eléctrica tienen una participación mucho menor. En este trabajo solo consideraremos cogeneración con motores de gas. El máximo rendimiento energético de un modulo de cogeneración está limitado por la cantidad de calor recuperable del motor pero también por el nivel de temperatura exigido (calidad). El sistema de recuperación se configurará teniendo en cuenta las características de la demanda de calor y la conveniencia de utilizar uno o varios niveles térmicos. Se consiguen mayores rendimientos cuando se recupera calor a un solo nivel de baja temperatura (Ramos, 2003). 


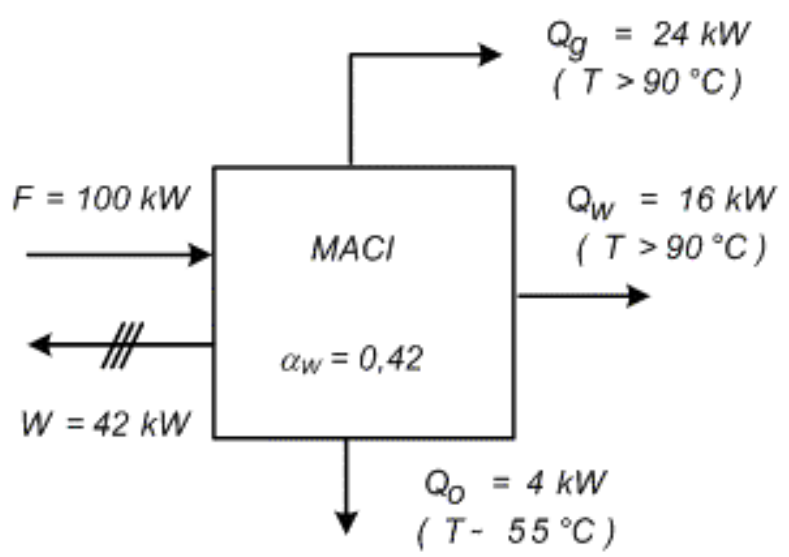

Fig. 3: Cogeneración con MACI

La Fig. 3, muestra las prestaciones de los módulos de cogeneración basados en una serie de motores alternativos de combustión interna a gas (MACl) con potencia nominal mayor que $1000 \mathrm{~kW}$ (Lozano et al., 2004a). EI MACl "promedio" que tiene un rendimiento $\alpha_{w}=0,42$ consume $100 \mathrm{~kW}$ de combustible para producir $42 \mathrm{~kW}$ de trabajo, siendo posible recuperar calor en las siguientes cantidades y niveles de temperatura: i) $4 \mathrm{~kW}\left(Q_{o}\right)$ como agua caliente a $t \approx 55^{\circ} \mathrm{C}$ desde el circuito de refrigeración del aceite de lubricación; ii) $16 \mathrm{~kW}\left(Q_{w}\right)$ como agua caliente a $t>90^{\circ} \mathrm{C}$ desde el circuito de refrigeración de los cilindros del motor; y iii) a partir de los gases de escape $\left(Q_{g}\right)$, recuperando 24 $\mathrm{kW}$ como agua caliente a $t>90^{\circ} \mathrm{C}$.

Suponiendo que se aprovecha todo el calor recuperable del motor $Q=Q_{g}+Q_{w}+Q_{o}=44 \mathrm{~kW}$, resulta un sistema de cogeneración con $\alpha_{Q}=0,44, \eta=0,86$ y $\beta \approx 1,05$. Suponiendo, además, que al instalar el sistema se evitará la producción eléctrica $W$, con centrales de ciclo combinado de rendimiento $\eta_{W}=$ 0,55 , y la producción de calor $Q$, con una caldera de gas de rendimiento $\eta_{Q}=0,90$, puede estimarse un ahorro de gas natural del $20 \%(I A F \approx 0,20)$ y un rendimiento eléctrico equivalente del $82 \%\left(\eta_{\text {eq }}=\right.$ $0,82)$. En las Figs. 4 y 5 , se muestran gráficamente las prestaciones de este sistema.

En la Fig. 6, se presenta el esquema de un sistema simple de cogeneración indicando los flujos de energía más importantes. El centro consumidor plantea una demanda de calor $Q_{D}$ y trabajo $E_{D}$ que debe ser atendida con el calor y trabajo cogenerados $W_{C}$ y $Q_{C}$ o de modo convencional (energía eléctrica comprada $E_{C}$ y calor producido en la caldera $Q_{A}$ ). En general, el sistema podrá vender energía eléctrica $E_{V}$ y despilfarrar calor al ambiente $Q_{L}$ si produce excedentes.

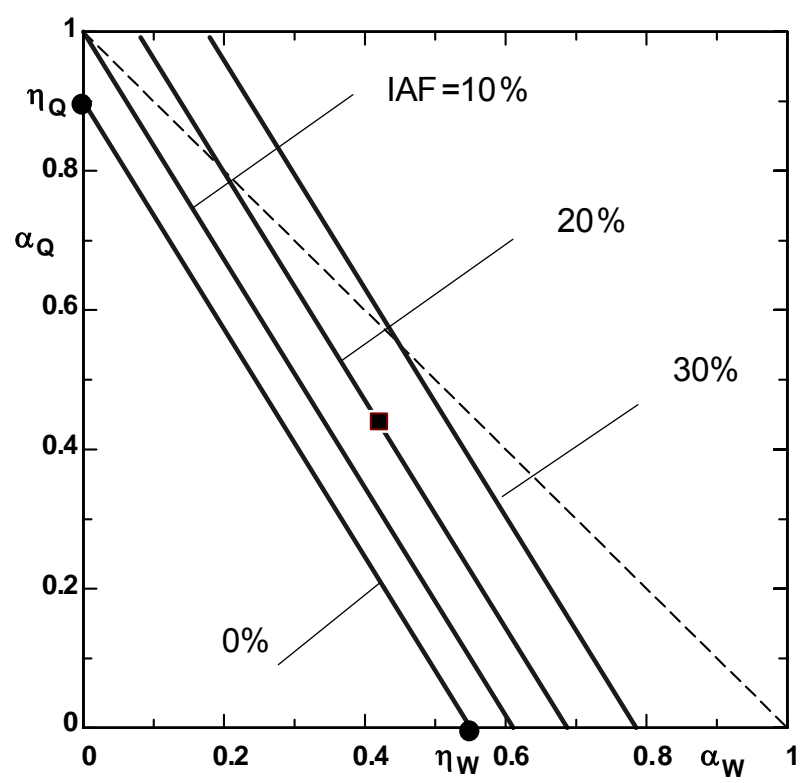

Fig. 4: Ahorro de energía

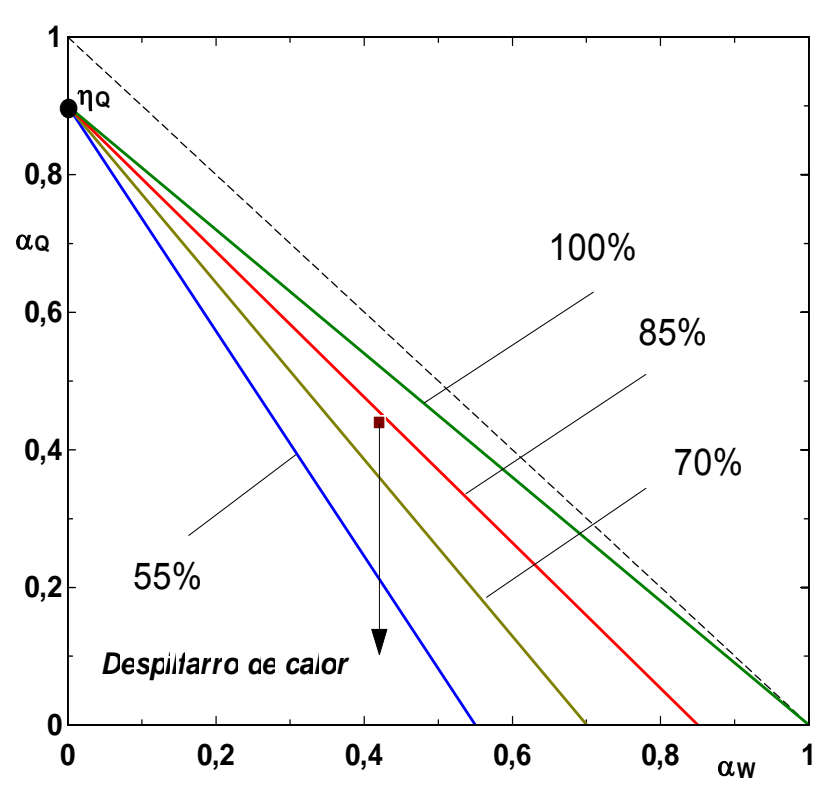

Fig. 5: Rendimiento eléctrico equivalente 


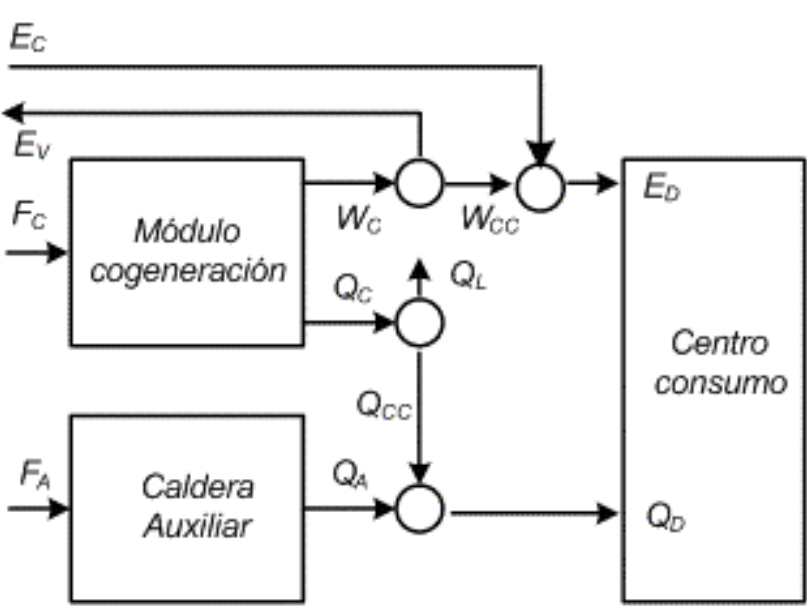

Fig. 6: Sistema simple de cogeneración

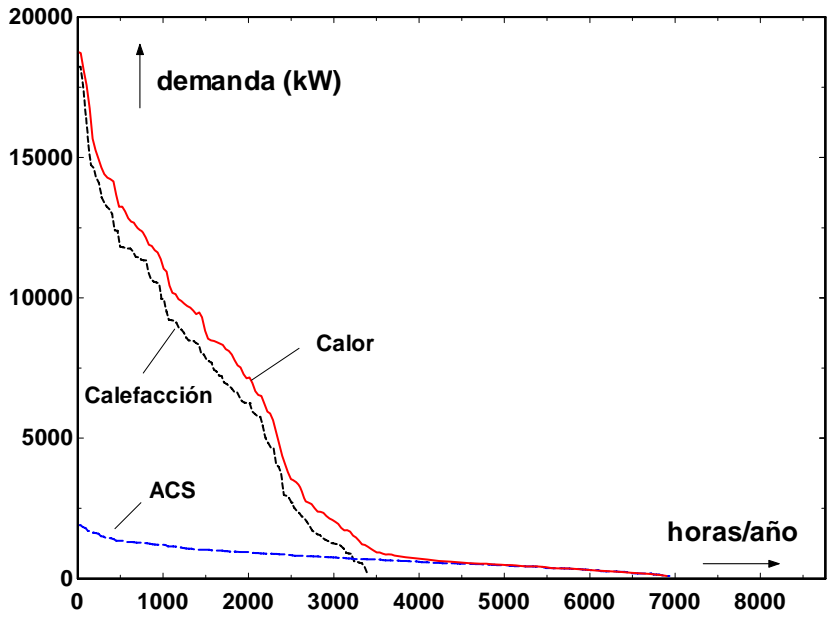

Fig. 7: Curvas monótonas de demanda

\section{DEMANDA}

La demanda energética de los edificios tiene las características siguientes: i) consumo de varias formas de energía (electricidad, calor, etc.) para atender los diferentes servicios (climatización, ACS, iluminación, electrodomésticos, ofimática, etc.); ii) concentración de la demanda de determinados servicios (climatización) en algunos meses del año; y iii) fuerte variación temporal de los consumos por factores ambientales y de ocupación. Es importante disponer de información lo más completa posible sobre la demanda energética para conseguir el mejor diseño de la planta de servicios. Existen distintos modos de presentar los datos de demanda: i) utilizando valores puntuales que caracterizan la potencia máxima y el consumo específico anual (p.e. $0,2 \mathrm{~kW} / \mathrm{m}^{2}$ y $50 \mathrm{kWh} /\left(\mathrm{m}^{2}\right.$ año)); ii) mediante curvas de duración anual de la demanda (ver Fig. 7) conocidas también como curvas monótonas de demanda; y iii) consumos "hora-por-hora" a lo largo del año. El primer modo aporta muy poca información y sirve únicamente en caso de instalaciones simples sobre las que se dispone de gran experiencia. El segundo modo, que es el que se utiliza aquí, sirve para tomar decisiones sobre que tipo de equipos emplear para satisfacer la demanda (y la capacidad a instalar) y para valorar distintas estrategias de operación. El tercer modo aporta sin duda la información más completa pero en muchos casos resulta difícil de conseguir y no permite un análisis generalizado.

En el presente trabajo, se utilizan las curvas de duración anual de la demanda de la Fig. 7, para estimar el tamaño de los equipos a instalar en una planta de cogeneración para un complejo residencial de 5000 viviendas ubicado en Zaragoza (España). La curva de duración anual representa el número de horas en el año (eje de abscisas) con una demanda mayor o igual que la potencia térmica indicada en el eje de ordenadas. La curva monótona de calefacción de la Fig. 7, indica que el complejo residencial: i) tiene una demanda pico de calor de $\sim 18 \mathrm{MW}$; ii) demanda calefacción durante $\sim 3500$ horas al año, iii) la demanda supera los $\sim 5 \mathrm{MW}$ durante 2200 horas al año, etc. Para construir una nueva curva monótona que exprese aproximadamente el efecto combinado de varias demandas de calor puede procederse como sigue: i) representar las curvas monótonas de todas las demandas de calor en la misma gráfica; ii) si las demandas de calor son simultáneas, la curva compuesta se obtiene sumando verticalmente las demandas correspondientes al mismo número de horas; y si las demandas de calor son asíncronas la curva compuesta se obtiene sumando horizontalmente las horas correspondientes a la misma demanda. Como se aprecia en la Fig. 7, la curva monótona de Calor resultante de agregar las demandas de Calefacción y ACS responde aproximadamente a lo indicado para el caso de demandas simultáneas.

A partir de la demanda anual de energía térmica $E Q D_{\text {año }}\left[\mathrm{kWh} / \mathrm{año}\right.$, de su duración $H_{\max }[\mathrm{h} / \mathrm{año}$ y de su potencia máxima $Q D_{\max }[\mathrm{kW}]$, la curva monótona de demanda puede aproximarse por el modelo simple $Q_{D}=a \cdot \exp (-b \cdot H)$, donde $a$ y $b$ son coeficientes de ajuste. Un método para determinar los coeficientes consiste en imponer la condición de potencia térmica máxima $a=Q D_{\max }$ y calcular $b$ de modo que se cumpla la condición de demanda anual de energía térmica $E Q D_{\text {año }}=\int_{0 \rightarrow H \text { máx }} Q_{D}(H) d H$. Para la demanda de calor de la Fig. 7, correspondiente al complejo residencial: $E Q D_{\text {año }}=29054 \cdot 10^{3}$, $H_{\max }=6935, Q D_{\max }=a=18762$ y $b=0,000638$. La representación gráfica puede verse en la Fig. 8. 


\section{EVALUACION ECONOMICA}

Dos decisiones importantes a tomar en el diseño de un sistema de cogeneración son la potencia eléctrica a instalar $W_{\text {ins }}[\mathrm{kW}]$ en módulos de cogeneración y la política a seguir para operar el sistema. Ambas decisiones van ligadas. Un análisis preliminar basado en los criterios de eficiencia antes presentados y en los criterios operacionales que explicamos a continuación facilitará la tarea.

\section{Criterios operacionales}

El factor de utilización de la potencia instalada en módulos de cogeneración viene dado por

$$
F U W=\left(\int_{0}^{H o p e} W_{c}(H) d H\right) /\left(8760 W_{\text {ins }}\right)
$$

donde $W_{c}[\mathrm{~kW}] \leq W_{\text {ins }}$ es el trabajo cogenerado a lo largo de la operación anual de duración Hope [h]. La tasa de cobertura es la fracción del consumo de calor anual atendido por cogeneración, o sea

$$
T C Q=\left(\int_{0}^{H o p e} Q_{c c}(H) d H\right) / E Q D_{a \tilde{n} o}
$$

donde $Q_{c c}[\mathrm{~kW}]$ es el calor recuperado y posteriormente consumido (no despilfarrado, ver Fig. 6). El grado de aprovechamiento del calor cogenerado puede expresarse en función de los parámetros anteriores y de la característica $\beta$ de los módulos de cogeneración empleados

$$
G A C=\frac{\int_{0}^{H o p e} Q_{C C}(H) d H}{\int_{0}^{H o p e} Q_{C}(H) d H} \approx \frac{T C Q}{F U W} \frac{E Q D_{\text {año }}}{8760 W_{\text {ins }} \beta}
$$

Idealmente, el mejor diseño del sistema de cogeneración conseguirá a la vez una tasa de cobertura elevada (mayor contribución de la cogeneración a la atención de la demanda de calor), un grado de aprovechamiento elevado (mayor eficiencia energética) y un factor de utilización elevado (rápida recuperación de la inversión realizada). Las curvas monótonas de demanda son muy útiles, pues si conocemos la potencia eléctrica o térmica de los módulos de cogeneración y los modos de regulación de carga permitidos podemos estimar fácilmente los parámetros anteriores y realizar una estimación rápida de la viabilidad de la cogeneración.

\section{Ahorro económico y restricciones legales}

El balance que expresa el ahorro económico anual $A$ [\$/año] que supone la cogeneración es

$$
A=W_{i n s} 8760 \cdot F U W\left(p_{e}+\beta \cdot G A C \frac{p_{f a}}{\eta_{C}}-\frac{p_{f m}}{\alpha_{W}}\right)
$$

donde $p_{e}, p_{f a}$ y $p_{f m}[\$ / \mathrm{kWh}$ ] son los precios de la electricidad, del combustible de la caldera auxiliar y del combustible del modulo de cogeneración, respectivamente. Para maximizar el ahorro anual conviene instalar mucha potencia de cogeneración $\left(W_{\text {ins }} \uparrow\right)$ y utilizarla al máximo (FUW $\left.\uparrow\right)$. Pero también conviene aprovechar al máximo el calor cogenerado (GAC $\uparrow$ ). En la mayor parte de los casos, dichos intereses son contrapuestos y, además, deben tenerse en cuenta las limitaciones legales a satisfacer para entregar energía eléctrica a la red (Cardona y Piacentino, 2005). La legislación de España exige un rendimiento eléctrico equivalente mínimo del $55 \%$ en cogeneración con motores de gas, supuesto fijo $\eta_{Q^{*}}=0,9$.

$$
R E E=\frac{\int_{0}^{\text {Hope }} W_{c}(H) d H}{\int_{0}^{\text {Hope }}\left(F_{c}(H)-Q_{c c}(H) / \eta_{Q^{*}}\right) d H} \geq 0,55
$$




\section{Estrategias de operación}

Dos políticas límite de operación razonables son: i) operar a plena carga los módulos de cogeneración instalados y ii) ajustar la carga de operación de los módulos a la demanda de calor. En la segunda, el grado de aprovechamiento del calor será máximo pero el factor de utilización del motor quedará limitado. En la primera el factor de utilización podrá aumentarse, dentro de ciertos límites, pero a costa de un menor aprovechamiento del calor cogenerado. La toma de partido por una u otra no solo depende de factores económicos y normativos sino también de criterios como la facilidad de operación, la programación del mantenimiento de los motores, etc. Por otro lado debe tenerse en cuenta que el análisis aquí realizado supone que los precios de compra y venta de la energía eléctrica son iguales y constantes en el tiempo, lo cual no es del todo verosímil.

Según la Ec. 12, cuando $p_{e} \geq p_{f m} / \alpha_{W}$ el precio de la electricidad ya compensa el coste del combustible utilizado para producirla, aunque se despilfarre todo el calor cogenerado, y por tanto la operación del motor a plena carga y el máximo tiempo posible resulta lo más adecuado. Para operación a plena carga el ahorro económico anual puede reescribirse como

$$
A=W_{\text {ins }} \cdot \text { Hope }\left(p_{e}-\frac{p_{f m}}{\alpha_{W}}\right)+T C Q \cdot E Q D_{a \tilde{o} o} \frac{p_{f a}}{\eta_{C}}
$$

El segundo término de esta ecuación expresa el ahorro proveniente del combustible que se deja de consumir en la caldera auxiliar. Como puede apreciarse en la Fig. 8, una vez Hope > HA parte del calor producido por el motor a plena carga deberá despilfarrarse pues no hay demanda suficiente. Al aumentar Hope, TCQ aumenta aunque GAC disminuya (ver Fig. 9) y por lo tanto el segundo término continuará aumentando hasta alcanzar el límite $H C=H \max$. Con Hope $>H$ max el segundo término ya no aumenta pero el primero si que lo hace por lo que aún convendría operar el motor el máximo número de horas posible. Pero suele aparecer un límite cuando se requiere vender energía a la red. Por ejemplo, en el caso analizado se exige que el rendimiento eléctrico equivalente correspondiente a la operación anual supere el valor límite $\eta_{W^{*}}$ para alcanzar la condición de autogenerador. Esta condición también puede expresarse como

$$
\operatorname{GAC}(H) \geq \frac{\eta_{Q^{*}}}{\beta}\left(\frac{1}{\alpha_{W}}-\frac{1}{\eta_{W^{*}}}\right)
$$

La condición de un rendimiento eléctrico equivalente límite del 55\% suele alcanzarse con un número de horas de funcionamiento de los motores a plena carga $H B$ cumpliendo $H A<H B<H C$ (véanse las Figs. 8 y 9). En nuestro caso se alcanza cuando $G A C(H B)=0,4835$, lo que significa que solo se permite despilfarrar un poco más del $50 \%$ del calor cogenerado.

Como puede verse en la Fig. 10, con la política de operación de modular la carga de los motores, no despilfarrando calor, se consigue un rendimiento eléctrico equivalente elevado pero con un factor de utilización reducido que dificulta recuperar la inversión. Las Figs. 11, 12 y 13 muestran, respectivamente, la tasa de cobertura de la demanda de calor, el factor de utilización del motor y el grado de aprovechamiento del calor cogenerado para tres estrategias de operación con los motores a plena carga: A) máxima duración $H A$ compatible con un aprovechamiento total del calor, B) máxima duración $H B$ compatible con la condición de rendimiento eléctrico equivalente límite, y C) máxima duración de la demanda de calor Hmax; y una cuarta estrategia D) en la que la potencia eléctrica producida se modula de modo que el calor cogenerado atienda justo la demanda de calor. En abscisas se representa el cociente entre la potencia térmica aprovechable de los módulos de cogeneración $Q_{i n s}=\beta W_{\text {ins }}$ relativa a la demanda instantánea máxima de calor $Q D_{\max }$. Se representan con puntos (para las estrategias B y D) los resultados correspondientes a instalar 2, 3 y 4 motores de 2928 kW de potencia eléctrica nominal. Instalar 2 motores y operar con la estrategia B permite una alta cobertura de la demanda de calor $(\sim 70 \%)$ a la par que un factor de utilización elevado $(\sim 75 \%)$. Con 3 y 4 motores la tasa de cobertura sube pero a costa de reducir el factor de utilización. La estrategia D (modulación de carga) conduce siempre a una baja utilización de los motores. 
Un análisis detallado (Lozano et al., 2004b) da como solución óptima instalar 3 motores y operar con la estrategia $\mathrm{B}$, conjugando una alta cobertura $(\sim 80 \%)$ con un factor de utilización razonable $(\sim 60 \%)$.

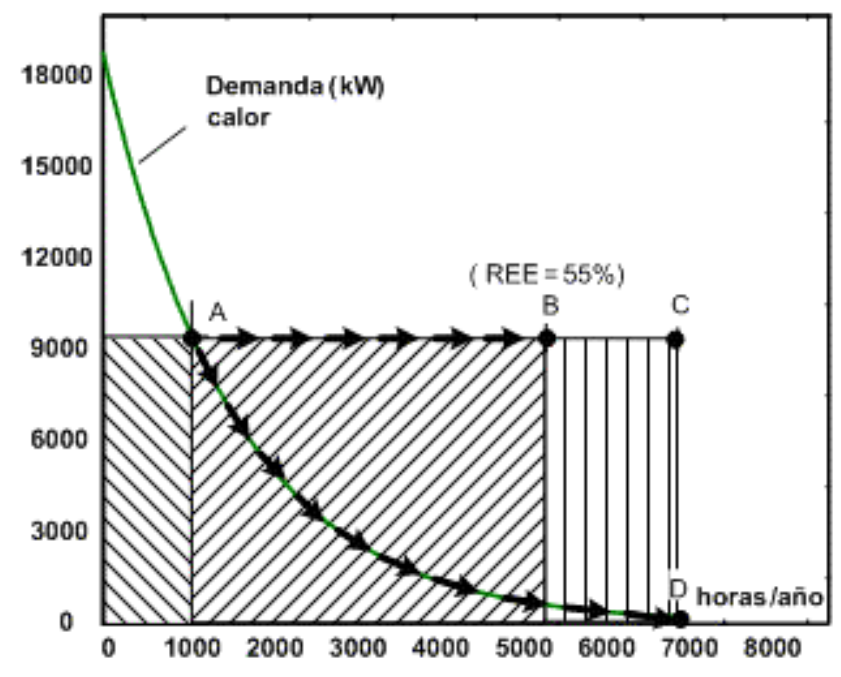

Fig. 8: Estrategias de operación

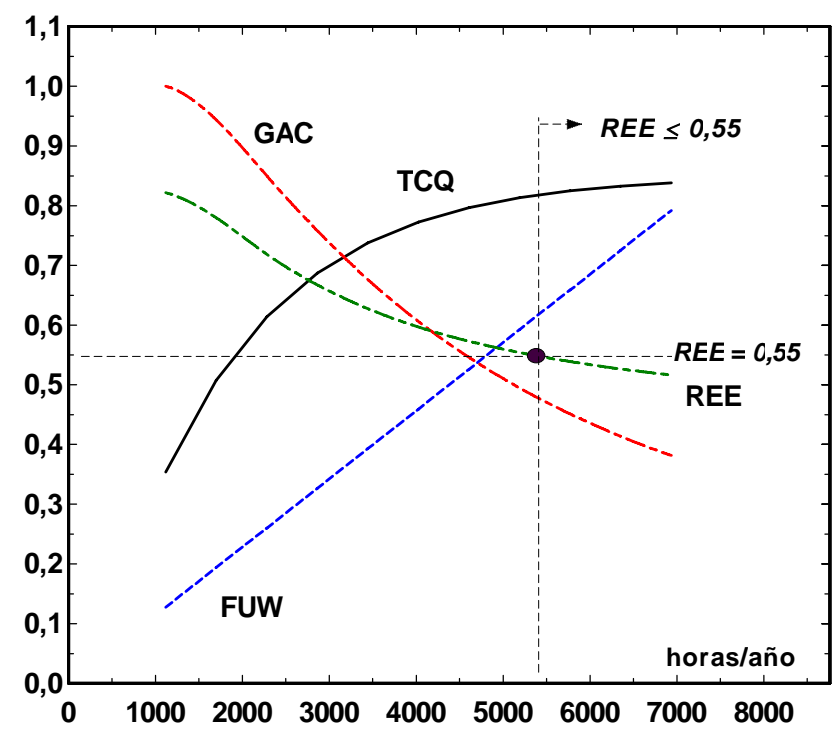

Fig. 9: Operación del motor a plena carga

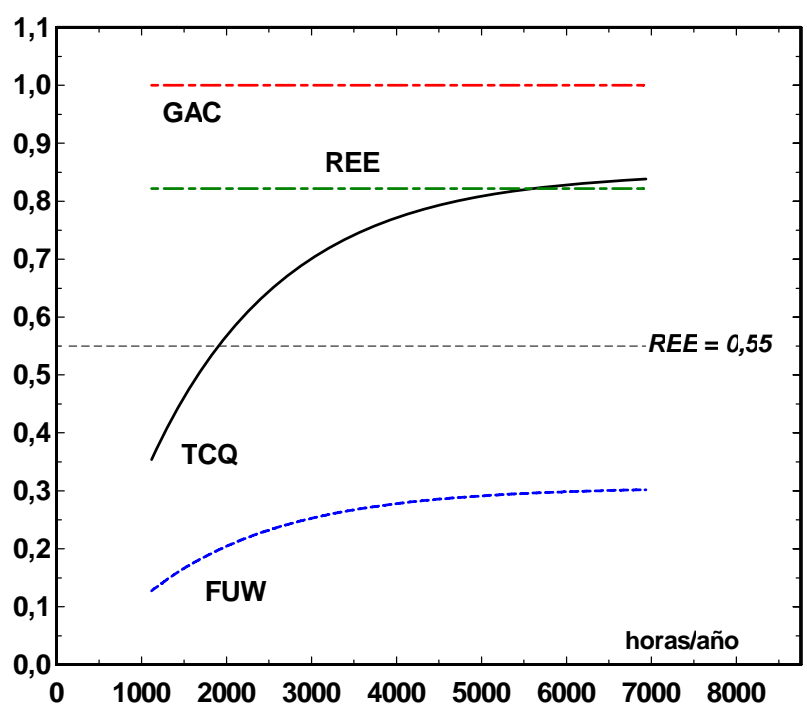

Fig. 10: Operación del motor modulando carga

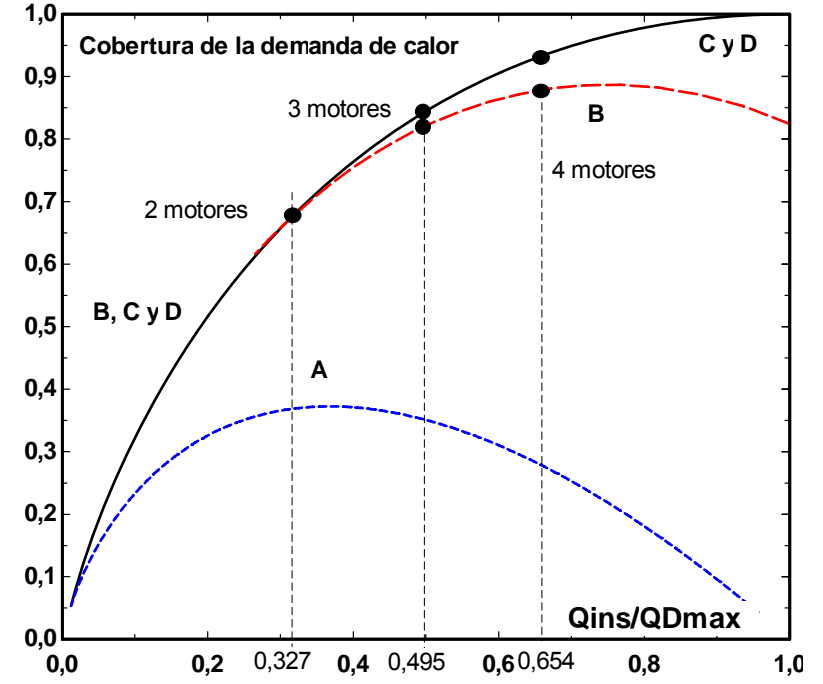

Fig. 11: Cobertura de la demanda

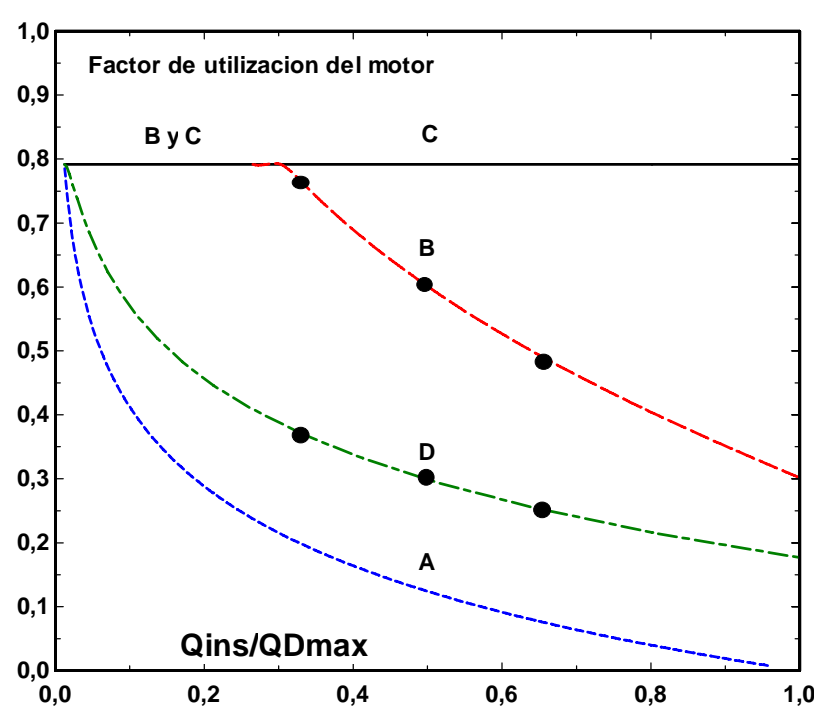

Fig. 12: Factor de utilización del motor

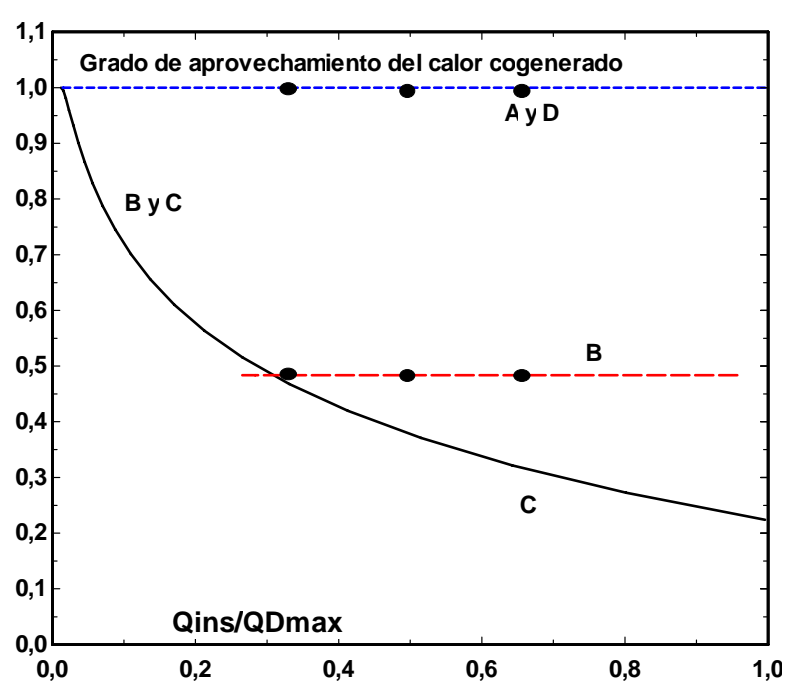

Fig. 13: Aprovechamiento del calor 


\section{CONCLUSIONES}

Los criterios tradicionales (ahorro de energía, rendimiento eléctrico equivalente, ahorro económico, etc.) que en cogeneración industrial se calculan fácilmente, dada la operación regular de los equipos a carga nominal, también pueden estimarse sin dificultad (incluso gráficamente) en cogeneración para edificios, a partir de las características técnicas y económicas de los módulos de cogeneración a emplear y de las curvas monótonas de demanda de calor. Un análisis detallado de cómo evolucionan los criterios operacionales definidos (factor de utilización, cobertura y grado de aprovechamiento) facilitará la decisión sobre que capacidad instalar y la estrategia de operación a emplear.

\section{AGRADECIMIENTOS}

Este trabajo ha sido financiado por el Plan Nacional Español de I+D+| 2000-2003 a través del Proyecto DPI 2003-00603.

\section{REFERENCIAS}

Cardona, E. y A. Piacentino; A methodology for sizing a trigeneration plant in Mediterranean areas, Applied Thermal Engineering, 23, 1665-1680 (2003).

Cardona, E. y A. Piacentino; Cogeneration: a regulatory framework toward growth, Energy Policy, 33, 2100-2111 (2005).

IEA; Energy Technology Perspectives - Scenarios \& Strategies to 2050, IEA (2006).

$\mathrm{Li}, \mathrm{H}$. y otros 3 autores; Energy utilization evaluation of CCHP systems, Energy and Buildings, 38, 253-257 (2006).

Lozano, M.A.; Diseño óptimo de sistemas simples de cogeneración, Información Tecnológica, 12(4), 3-58 (2001).

Lozano, M.A.; J. Ramos y R. Monzón, Análisis termoeconómico de sistemas de trigeneración, Anales de Ingeniería Mecánica, 15(2), 1341-1349 (2004a).

Lozano, M.A., J. Ramos y R. Monzón; Optimización de sistemas de cogeneración para calefacción y refrigeración de distrito, Anales de Ingeniería Mecánica, 15(2), 1385-1393 (2004b).

Lucas, K.; On the thermodynamics of cogeneration, Int. J. Therm. Sci., 39, 1039-1046 (2000).

Martens, A.; The energetic feasibility of CHP compared to the separate production of heat and power, Applied Thermal Engineering, 18, 935-946 (1998).

Petchers, N.; Combined heating, cooling and power handbook: technologies and applications, The Fairmont Press (2003).

Ramos, J.; Integración térmica de plantas de cogeneración y trigeneración, III Jornadas de Ingeniería Termodinámica, 653-651, Valencia-España (2003).

Tchouate, P.M y L. Bolle; Economie d'énergie en trigénération, International Journal of Thermal Sciences, 41, 1151-1159 (2002).

TriGeMed; Survey Report: Promotion of tri-generation technologies in the tertiary sector in Mediterranean countries, www.trigemed.com (2003).

Yokohama, R. y otros 3 autores; Development of a General-Purpose Optimal Planning System for Energy Supply Plants, ASME Journal of Energy Resources Technology, 116, 290-296 (1994). 